\title{
OPTIMALITY OF PRINCIPAL COMPONENT FILTER BANKS FOR DISCRETE MULTITONE COMMUNICATION SYSTEMS
}

\author{
P. P. Vaidyanathan ${ }^{\dagger}$, Yuan-Pei Lin ${ }^{\ddagger}$, Sony Akkarakaran ${ }^{\dagger}$, and See-May Phoong* \\ ${ }^{\dagger}$ Dept. Electrical Engr., Caltech, Pasadena, CA 91125 \\ $\ddagger$ Dept. of Elec. and Control Engr., National Chiao Tung Univ., Hsinchu, Taiwan \\ *Dept. Electrical Engr., National Taiwan University, Taipei, Taiwan.
}

\begin{abstract}
Discrete multitone modulation is an attractive method for communication over a non flat channel with possibly colored noise. The uniform DFT filter bank and cosine modulated filter bank have in the past been used in this system because of low complexity. We show in this paper that principal component filter banks, which are known to be optimal for data compression and denoising applications, are also optimal for a number of criteria in DMT communication.
\end{abstract}

\section{INTRODUCTION}

Figure 1 shows a maximally decimated analysis/synthesis system traditionally used in subband coding and more recently in signal denoising (all notations are as in [18]). A dual of this system called the transmultiplexer circuit is shown in Fig. 2. This is commonly used for conversion between time domain and frequency domain multiplexing. More recently this system has found application in the digital implementation of multicarrier systems, more popularly known as the DMT (discrete multitone) modulation systems. In this paper we will primarily be concerned with this application. Either of the systems shown in the two figures is said to be a biorthogonal system if the filters are such that

$$
\left.H_{k}\left(e^{j \omega}\right) F_{m}\left(e^{j \omega}\right)\right|_{\downarrow M}=\delta(k-m)
$$

This is equivalent to the perfect reconstruction property, that is, $\widehat{x}(n)=x(n)$ for all $n$ in Fig. 1, and

$$
y_{k}(n)=x_{k}(n)
$$

for all $k, n$ in Fig. 2. The set of $M$ filters $\left\{F_{k}(z)\right\}$ is said to be orthonormal if $\left.F_{k}\left(e^{j \omega}\right) F_{m}^{*}\left(e^{j \omega}\right)\right|_{\downarrow M}=$ $\delta(k-m)$ (equivalently the polyphase matrix is paraunitary [18]). In this case biorthogonality or perfect

\footnotetext{
${ }^{1}$ Work supported in parts by the ONR grant N00014-99-11002, USA, by Microsoft Research, Redmond, WA, and by the NSC 88-2218-E-009-016 and 88-2213-E-002-080, Taiwan, ROC.
}

reconstruction is achieved by the choosing $H_{k}\left(e^{j \omega}\right)=$ $F_{k}^{*}\left(e^{j \omega}\right)$.

The use of filter bank theory in the optimization of DMT systems has been of some interest in the past [10], [11]. In this paper we will show using the results of [3] that the principal component filter bank, which is known to be optimal for several problems involving the subband coder, will also be optimal in many respects for the DMT communications system.

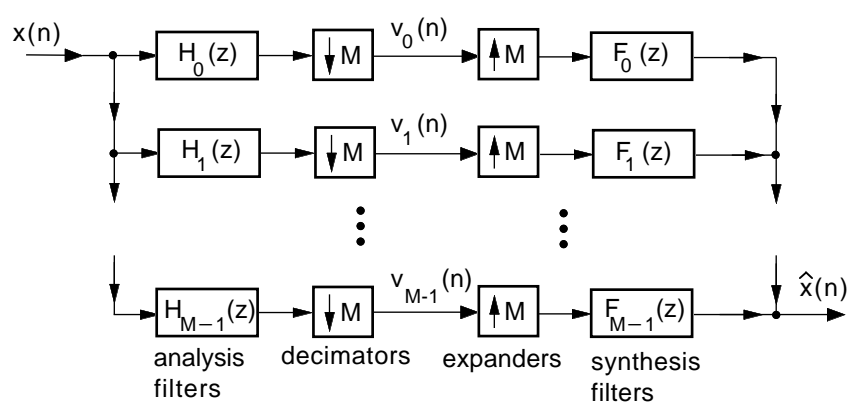

Figure 1. The subband coder system.

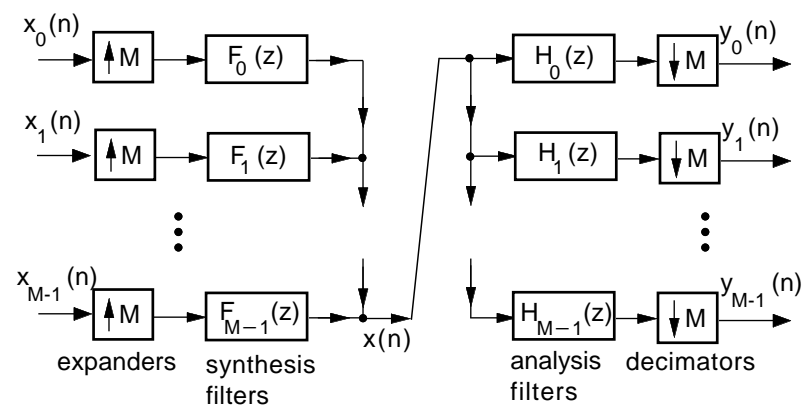

Figure 2. The digital transmultiplexer.

\section{PRINCIPAL COMPONENT FILTER BANKS}

The optimality of principal component filter banks (PCFBs) in the context of progressive transmission and 
subband coding was observed to various degrees by a number of authors $[2,12,15,17,19]$. To define a PCFB first consider two sets of $M$ nonnegative numbers $\left\{a_{n}\right\}$ and $\left\{b_{n}\right\}$. We say that $\left\{a_{n}\right\}$ majorizes $\left\{b_{n}\right\}$ if, after reordering such that $a_{n} \geq a_{n+1}$ and $b_{n} \geq b_{n+1}$, we have

$$
\sum_{n=0}^{P} a_{n} \geq \sum_{n=0}^{P} b_{n}
$$

for $0 \leq P \leq M-1$, with equality for $P=M-1$. Thus all the partial sums in $\left\{a_{n}\right\}$ dominate those in $\left\{b_{n}\right\}$. Consider a given class $\mathcal{C}$ of $M$-band uniform orthonormal filter banks. This class can be the class $\mathcal{C}_{t c}$ of transform coders (filter lengths $\leq M$ ), or the class $\mathcal{C}_{\text {ideal }}$ of ideal filter banks (filters allowed to have infinite order, like brickwall filters). Or it could be a practically attractive class like the FIR class $\mathcal{C}_{\text {fir }}$ with filter orders bounded by a fixed integer, or the cosine modulated class $\mathcal{C}_{c m f b}$. Given such a class $\mathcal{C}$ and an input power spectrum $S_{x x}\left(e^{j \omega}\right)$ we say that a filter bank $\mathcal{F}$ in $\mathcal{C}$ is a principal component filter bank or PCFB if the set $\left\{p_{k}^{2}\right\}$ of its subband variances (i.e., variances $\sigma_{v_{k}}^{2}$ of the signals $v_{k}(n)$ in Fig. 1) majorizes the set $\left\{q_{k}^{2}\right\}$ of subband variances of all other filter banks in the class. That is, with $p_{n}^{2} \geq p_{n+1}^{2}$ and $q_{n}^{2} \geq q_{n+1}^{2}$,

$$
p_{0}^{2} \geq q_{0}^{2}, p_{0}^{2}+p_{1}^{2} \geq q_{0}^{2}+q_{1}^{2}, \ldots
$$

and so forth. The equality $\sum_{k=0}^{M-1} p_{k}^{2}=\sum_{k=0}^{M-1} q_{k}^{2}$ follows automatically from orthonormality.

The advantage of PCFBs is that they are optimal for several problems. This includes subband coding with arbitrary (not necessarily high) bit rates, the denoising problem, and so forth, as elaborated in [3]. These arise from the result (proved in [3]) that any concave function $\phi$ of the subband variance vector

$$
\mathbf{v}=\left[\begin{array}{llll}
\sigma_{v_{0}}^{2} & \sigma_{v_{1}}^{2} & \ldots & \sigma_{v_{M-1}}^{2}
\end{array}\right]^{T}
$$

is minimized by a PCFB when one exists. Using this we show in this paper that PCFBs also serve as optimal solutions to certain problems in communication systems which use DMT modulation. It possible that PCFBs do not exist for certain classes but when they exist, they have the stated optimality. Whenever we say that the PCFB is optimal for a problem, the implicit assumption is that the class of filter banks searched is such that a PCFB exists.

For the transform coder class $\mathcal{C}_{t c}$, the $M \times M$ KLT of the input serves as the PCFB. For the ideal filter bank class $\mathcal{C}_{\text {ideal }}$, there is a systematic method to construct a PCFB by designing a sequence of compaction filters [19]. For example the filter bank in Fig. 3(b) is a PCFB for the power spectrum in Fig. 3(a).

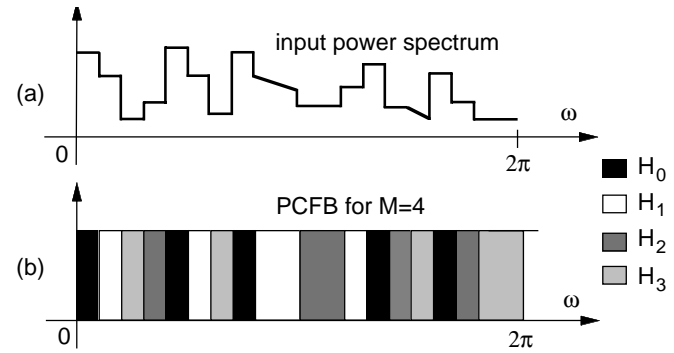

Figure 3. A power spectrum and its $\operatorname{PCFB}(M=4)$.

\section{THE DMT COMMUNICATION SYSTEM}

Figure 4 shows the essentials of discrete multitone communication. Background material on the DMT system and more generally on the use of digital filter banks in communications can be found in $[1,4,5,7,8,16]$.

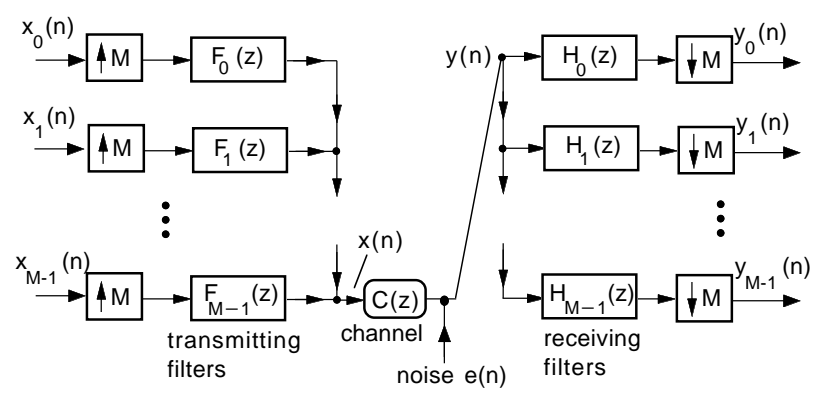

Figure 4. The discrete multitone communication system.

Briefly, here is how the system works: the signals $x_{k}(n)$ are $b_{k}$-bit symbols obtained from a PAM or QAM constellation [13]. Together these signals represent $\sum_{k} b_{k}=b$ bits, and are obtained from a $b$-bit block of a binary data stream [4]. The symbols $x_{k}(n)$ are then interpolated $M$-fold by the filters $F_{k}(z)$. Typically the filters $\left\{F_{k}\left(e^{j \omega}\right)\right\}$ constitute an orthonormal filter bank and their passbands cover different uniform regions of digital frequency $0 \leq \omega \leq 2 \pi$. The outputs of $F_{k}(z)$ can be regarded as modulated versions of the symbols. These are packed into $M$ adjacent frequency bands (passbands of the filters) and added to obtain the composite signal $x(n)$. This is then sent through the channel which is represented by a transfer function $C(z)$ and additive Gaussian noise $e(n)$ with power spectrum $S_{e e}\left(e^{j \omega}\right)$. In actual practice the channel is a continuous-time system preceded by $D / A$ conversion and followed by $A / D$ conversion. We have replaced this with discrete equivalents $C(z)$ and $e(n)$.

The received signal $y(n)$ is a distorted and noisy version of $x(n)$. The receiving filter bank $\left\{H_{k}(z)\right\}$ separates this signal into the components $y_{k}(n)$ which are distorted and noisy versions of the symbols $x_{k}(n)$. The task at this point is to correctly detect the value of $x_{k}(n)$ from $y_{k}(n)$. There is a probability of error in 
this detection which depends on the signal and noise levels.

If the filter bank $\left\{F_{k}, H_{m}\right\}$ is biorthogonal then we have the perfect reconstruction property $y_{k}(n)=x_{k}(n)$ in absence of channel imperfections (i.e., assuming $C(z)=1$ and $e(n)=0)$. In practice we cannot assume this. We will assume that $\left\{F_{k}, H_{m}\right\}$ is biorthogonal (in fact orthonormal, see below) and that the receiving filters are

$$
H_{k}(z) / C(z)
$$

instead of $H_{k}(z)$, so that $C(z)$ is compensated or equalized completely.

\section{OPTIMAL DMT SYSTEMS}

For simplicity we assume that $x_{k}(n)$ are PAM symbols [13]. Assuming that $x_{k}(n)$ is a random variable with $2^{b_{k}}$ equiprobable levels, its variance represents the average power $P_{k}$ in the symbol $x_{k}(n)$. The Gaussain channel noise $e(n)$ is filtered through $H_{k}(z) / C(z)$ and decimated by $M$. For the purpose of variance calculation, the model for the noise $q_{k}(n)$ at the detector input can therefore be taken as in Fig. 5. Let $\sigma_{q_{k}}^{2}$ be the variance of $q_{k}(n)$. Then the probability of error in detecting the symbol $x_{k}(n)$ is [13]

$$
\mathcal{P}_{e}(k)=2\left(1-2^{-b_{k}}\right) \mathcal{Q}\left(\sqrt{\frac{3 P_{k}}{\left(2^{2 b_{k}}-1\right) \sigma_{q_{k}}^{2}}}\right)
$$

where $\mathcal{Q}(v) \triangleq \int_{v}^{\infty} e^{-u^{2} / 2} d u / \sqrt{2 \pi}$ (area of the normalized Gaussian tail).

\subsection{Minimizing Transmitted Power}

Since the $\mathcal{Q}$-function can be inverted for any nonnegative argument, we can invert (1) to obtain

$$
P_{k}=\beta_{k}\left(\mathcal{P}_{e}(k), b_{k}\right) \times \sigma_{q_{k}}^{2}
$$

where the exact nature of the function $\beta_{k}(.,$.$) is not$ of immediate interest. This expression says that if the probability of error has to be $\mathcal{P}_{e}(k)$ or less at the bit rate $b_{k}$, then the power in $x_{k}(n)$ has to be at least as large as $P_{k}$. The total transmitted power is therefore

$$
P=\sum_{k=0}^{M-1} P_{k}=\sum_{k=0}^{M-1} \beta_{k}\left(\mathcal{P}_{e}(k), b_{k}\right) \times \sigma_{q_{k}}^{2}
$$

Let us assume that the bit rates $b_{k}$ and probabilites of error $\mathcal{P}_{e}(k)$ are fixed. For this desired combination of $\left\{b_{k}\right\}$ and $\left\{P_{k}\right\}$, the total power required depends on the distribution of noise variances $\left\{\sigma_{q_{k}}^{2}\right\}$.

From Eq. (2) we see that the power $P_{k}$ in the $k$ th band is a linear (hence concave) function of $\sigma_{q_{k}}^{2}$. The total transmitted power $P$ is therefore a concave function of the noise variance vector

$$
\left[\begin{array}{llll}
\sigma_{q_{0}}^{2} & \sigma_{q_{1}}^{2} & \ldots & \sigma_{q_{M-1}}^{2}
\end{array}\right]^{T}
$$

From Fig. 5 we see that this is the vector of subband variances for the orthonormal filter bank $\left\{H_{k}\left(e^{j \omega}\right)\right\}$ in response to the power spectrum $S_{e e}\left(e^{j \omega}\right) /\left|C\left(e^{j \omega}\right)\right|^{2}$. Recalling the discussion of PCFB from Sec. 2 we now see that the orthonormal filter bank $\left\{H_{k}\left(e^{j \omega}\right)\right\}$ which minimizes total power for fixed error probabilities and bit rates is indeed a PCFB for the power spectrum

$$
S_{e e}\left(e^{j \omega}\right) /\left|C\left(e^{j \omega}\right)\right|^{2}
$$

(for whatever class $\mathcal{C}$ is under consideration). Having identified this $\mathrm{PCFB}$, the variances $\sigma_{q_{k}}^{2}$ are readily computed, from which the powers $P_{k}$ for fixed bit rate $b_{k}$ and error probabilty $\mathcal{P}_{e}(k)$ can be found (using (2)), and the minimized power $P$ calculated.

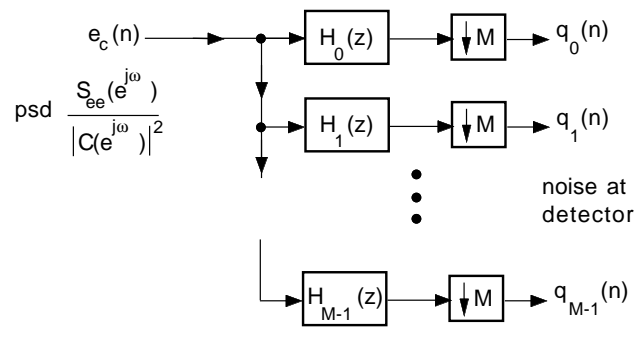

Figure 5. A model for noise at detector input.

\subsection{Maximizing Total Bit Rate}

Returning to the error probability expression (1) let us now invert it to obtain a formula for the bit rate $b_{k}$. This is tricky because of the way $b_{k}$ occurs in two places. The factor $\left(1-2^{-b_{k}}\right)$ however is a weak function of $b_{k}$ in the sense that it varies from 0.5 to 1 as $b_{k}$ changes from one to infinity. So we will replace $(1-$ $2^{-b_{k}}$ ) with unity. Then Eq. (1) yields

$$
b_{k}=0.5 \log _{2}\left(1+\frac{3}{\left[\mathcal{Q}^{-1}\left(\mathcal{P}_{e}(k) / 2\right)\right]^{2}} \frac{P_{k}}{\sigma_{q_{k}}^{2}}\right)
$$

so the total bit rate is

$$
b=0.5 \sum_{k=0}^{M-1} \log _{2}\left(1+\frac{3}{\left[\mathcal{Q}^{-1}\left(\mathcal{P}_{e}(k) / 2\right)\right]^{2}} \frac{P_{k}}{\sigma_{q_{k}}^{2}}\right)
$$

This is the bit rate achieved by the DMT system without channel coding, for fixed error probabilities $\left\{\mathcal{P}_{e}(k)\right\}$ and powers $\left\{P_{k}\right\}$. Since function

$$
\log _{2}\left(1+\frac{a}{x}\right)
$$

is convex in $x$ (for $a, x>0$ ), the total bit rate is convex in the variance vector (4). Thus the orthonormal filter bank $\left\{H_{k}\left(e^{j \omega}\right)\right\}$ which maximizes bit rate for fixed error probabilities and powers is again a PCFB for the same power spectrum $S_{e e}\left(e^{j \omega}\right) /\left|C\left(e^{j \omega}\right)\right|^{2}$ as before. This is very appealing since the maximization of 
bit rate and minimization of total power are consisent goals.

The preceding result is true regardless of how the total power $P=\sum_{k} P_{k}$ is allocated among the bands. In particular we can perform optimum power allocation. We have

$$
b=0.5 \sum_{k=0}^{M-1} \log _{2}\left(1+\frac{P_{k}}{N_{k}}\right)
$$

where $N_{k}=\sigma_{q_{k}}^{2}\left[\mathcal{Q}^{-1}\left(\mathcal{P}_{e}(k) / 2\right)\right]^{2} / 3$. The optimization of $\left\{P_{k}\right\}$ for fixed total power $P=\sum_{k} P_{k}$ is a standard problem in information theory [6]. The solution is given by the water pouring rule, described as

$$
P_{k}= \begin{cases}\lambda-N_{k} & \text { if this is nonegative } \\ 0 & \text { otherwise. }\end{cases}
$$

where $\lambda$ is chosen to meet the power constraint. See Fig. 6. This power allocation is optimal regardless of the exact choice of the filter bank $\left\{H_{k}(z)\right\}$. In particular if $\left\{H_{k}(z)\right\}$ is chosen as the optimal PCFB and then power is allocated as above, it provides the maximum possible DMT bit rate $b$ for fixed total power and fixed set of error probabilities.

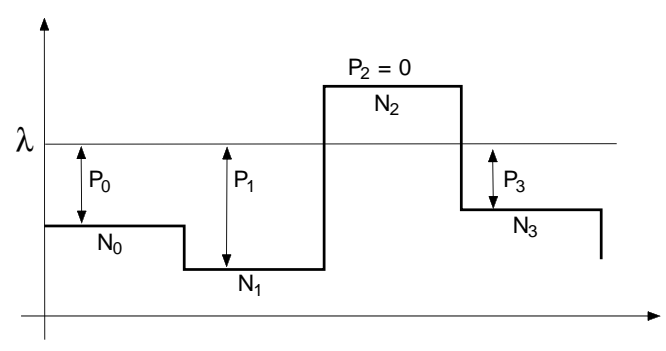

Figure 6. Optimal power allocation by water pouring.

\section{CONCLUDING REMARKS}

DMT systems based on fixed filter banks such as the DFT or cosine modulated filter banks [5, 14] are attractive because of the efficiency with which they can be implemented. A PCFB solution in general may not lead to such an efficient implementation, even though it is optimal from a performance point of view. The PCFB system serves as a useful bound for performance comparisons for fixed number of bands $M$. Such a comparison appears to be the next logical step for future work. If the performance gap between a practical system and the PCFB solution is small in a particular application, this gives the assurance that we are not very far from optimality.

\section{References}

[1] Akansu, A. N., Duhamel, P., Lin, X., and Courville, M. de. "Orthogonal transmultiplexers in communications: a review," IEEE Trans. SP, April 1998.
[2] Akkarakaran, S., and Vaidyanathan, P. P. "On optimization of filter banks with denoising applications", Proc. IEEE ISCAS, Orlando, FL, June 1999.

[3] Akkarakaran, S., and Vaidyanathan, P. P. "The role of principal component filter banks in noise reduction", Proc. SPIE, Denver, CO, July 1999.

[4] Bingham, J. A. C. "Multicarrier modulation for data transmission: an idea whose time has come," IEEE Comm. Mag., pp. 5-14, May 1990.

[5] Chow, J. S., Tu, J. C., and Cioffi, J. M. "A discrete multitone transreceiver system for HDSL applications," IEEE J. Selected Areas of Communications, pp. 895-908, Aug., 1991.

[6] Cover, T. M., and Thomas, J. A. Elements of information theory, John Wiley \& Sons, Inc., 1991.

[7] Kalet, I. "The multitone channel", IEEE Trans. Comm., pp. 119-124, Feb. 1989.

[8] Kalet, I. "Multitone modulation", in Subband and wavelet transforms, edited by Akansu, A. N., and Smith, M. J., Kluwer Academic Publishers, 1996.

[9] Koilpillai, R. D., Nguyen, T. Q., and Vaidyanathan, P. P. "Some results in the theory of cross talk free transmultiplexers," IEEE Trans. SP, Oct. 1991.

[10] Lin, X., and Akansu, A. N. "A distortion analysis and optimal design of orthonormal basis for DMT receivers", Proc. IEEE, ICASSP, pp. 1475-1478, 1996. [11] Lin, Y-P., and Phoong, S-M. "Optimal DMT transreceivers over fading channels", Proc. IEEE, ICASSP, pp. 1397-1400, Phoenix, AZ, 1999.

[12] Moulin, P., Anitescu, M., and Ramchandran, K. "Theory of rate-distortion optimal, constrained filter banks - applications to IIR and FIR biorthogonal designs," preprint, June 1998.

[13] Proakis, J. G. Digital communications, McGraw Hill 1995.

[14] Rizos, A. D., Proakis, J. G., and Nguyen, T. Q. "Comparison of DFT and cosine modulated filter banks in multicarrier modulation," Proc. of Globecom, pp. 687-691, Nov. 1994.

[15] Tsatsanis, M. K., and Giannakis, G. B., "Principal component filter banks for optimal multiresolution analysis," IEEE Trans. SP, Aug. 1995.

[16] Tzannes, M. A., Tzannes, M. C., Proakis, J. G., and Heller, P. N. "DMT systems, DWMT systems, and digital filter banks", Proc. ICC, pp. 311-315, 1994.

[17] Unser, M. "An extension of the KLT for wavelets and perfect reconstruction filter banks," Proc. SPIE, Wavelet Appl. in Signal and Image Proc., pp. 45-56, San Diego, CA, 1993.

[18] Vaidyanathan, P. P. Multirate systems and filter banks, Prentice Hall, Inc., 1993.

[19] Vaidyanathan, P. P. "Theory of optimal orthonormal subband coders," IEEE Trans. SP, June 1998.

[20] Vetterli, M. "Perfect transmultiplexers," Proc. ICASSP, pp. 2567-2570, 1986.

[21] Xuan, B., and Bamberger, R. H. "FIR principal component filter banks," IEEE Trans. SP, April 1998. 